\title{
Comparative Analysis of Reactant and Product Adsorption Energies in the Selective Oxidative Coupling of Alcohols to Esters on $\operatorname{Au}(111)$
}

\author{
Selma Şenozan ${ }^{1} \cdot$ Hande Ustunel $^{1} \cdot$ Mustafa Karatok $^{2} \cdot$ Evgeny I. Vovk $^{2,3}$. \\ Asad A. Shah ${ }^{2} \cdot$ Emrah Ozensoy $^{2} \cdot$ Daniele Toffoli $^{4}$
}

Published online: 22 July 2016

(c) Springer Science+Business Media New York 2016

\begin{abstract}
Gold-based heterogeneous catalysts have attracted significant attention due to their selective partial oxidation capabilities, providing promising alternatives for the traditional industrial homogeneous catalysts. In the current study, the energetics of adsorption/desorption of alcohols $\left(\mathrm{CH}_{3} \mathrm{OH} /\right.$ methanol, $\mathrm{CH}_{3} \mathrm{CH}_{2} \mathrm{OH} /$ ethanol, $\mathrm{CH}_{3}$ $\mathrm{CH}_{2} \mathrm{CH}_{2} \mathrm{OH} / n$-propanol) and esters $\left(\mathrm{HCOOCH}_{3} /\right.$ methyl formate, $\mathrm{CH}_{3} \mathrm{COOCH}_{3} /$ methyl acetate, and $\mathrm{CH}_{3} \mathrm{COOCH}_{2}$ $\mathrm{CH}_{3} /$ ethyl acetate) on a planar $\mathrm{Au}(111)$ surface was investigated in conjunction with oxidative coupling reactions by means of temperature programmed desorption (TPD) and dispersion-corrected density functional theory (DFT) calculations. The results reveal a complex interplay between inter-molecular and surface-molecule interactions, both mediated by weak van der Waals forces, which dictates their relative stability on the gold surface. Both experimental and theoretical adsorption/desorption energies of the investigated esters are lower than those of the alcohols from which they originate through oxidative coupling reactions. This result can be interpreted as an
\end{abstract}

Hande Ustunel

ustunel@metu.edu.tr

$\triangle$ Daniele Toffoli

toffoli@units.it

1 Department of Physics, Middle East Technical University, Dumlupinar Bulvari 1, 06800 Ankara, Turkey

2 Department of Chemistry, Bilkent University, 06800 Bilkent, Ankara, Turkey

3 Boreskov Institute of Catalysis, Novosibirsk, Russian Federation 630090

4 Dipartimento di Scienze Chimiche e Farmaceutiche, Università degli Studi di Trieste, Via L. Giorgieri 1, 34127 Trieste, Italy important indication in favor of the selectivity of Au surfaces in alcohol oxidative coupling/partial oxidation reactions, allowing facile removal of partial oxidation products immediately after their generation preventing their complete oxidation to higher oxygenates.

Keywords Density functional theory · van der Waals . Adsorption energy · Partial oxidation · Alcohols . Heterogeneous catalyst

\section{Introduction}

Concerted global efforts to cope with environmental and climate issues related to the world's large dependence on fossil fuels as energy vectors are focused on improving efficiencies of current energy conversion processes and transformation systems. In addition to their direct utilization as fuels, alcohols (particularly methanol and ethanol) have high $\mathrm{H} / \mathrm{C}$ atomic ratios rendering them convenient feeds for onboard hydrogen production. Furthermore, their higher reactivity (compared to alkanes) requiring milder conditions for the corresponding hydrogen production reactions make them attractive hydrogen carriers. Besides these catalytic routes converting methanol and ethanol to syngas, partial oxidation and oxidative coupling of alcohols are other fast developing research fields related to chemical energy efficiency and energy conversion. In view of these facts, alcohols also provide extremely promising energy alternatives that can be obtained in a sustainable fashion from biological feedstocks. During the oxidation of alcohols, a rich selection of industrially important intermediates is produced. Esters are probably the industrially more valuable products of partial oxidation of alcohols, while 
commercially less valuable oxygenates include aldehydes, ketones, and organic acids. [1-10].

Unique heterogeneous catalysts can be designed by exploiting the fundamental aspects of surface chemistry that selectively favor particular reaction pathways during the oxidation process over a multitude of others, yielding valuable products. Although gold surfaces in bulk form are well-known to suffer from low reactivity (typically due to the low adsorption energies of the corresponding reactants), they can be tailored at the nanometer scale to obtain catalytically active phases [11-13]. Thus, a significant portion of the recent activity in the design of heterogeneous catalysts to be used in the selective oxidation of alcohols has been directed towards gold-based materials [14-18]. Recent advances have made it possible to improve the reactivity of gold surfaces by means of oxygen pretreatment [10], alloying [19] and surface morphology or porosity modification at the nanometer scale [20].

It has been reported that hydrocarbons and numerous oxygenates, such as alcohols and aldehydes, predominantly adsorb on clean gold surfaces molecularly (non-dissociatively) and desorb in a reversible manner [10, 21-29]. A careful assessment of various types of intermolecular interactions as well as interactions between adsorbates and the underlying surface is crucial to provide a complete account of the energetics associated with such adsorption systems. Weak van der Waals interactions, typically ignored in the presence of covalent bonds, take center stage in determining the favorable adsorption sites on the catalyst surfaces. In addition to catalyst design, noncovalent interactions between organic molecules and planar single crystal metal surfaces have also been recently studied in the context of self-assembled monolayer (SAM) systems and identified as the principal factor in determining the assembly patterns [30-34].

A comprehensive understanding of the adsorption site preferences and adsorption strengths of the reactants and the intermediates involved in the complex reaction mechanisms is essential for predicting the catalytic selectivity of a particular catalyst in a given reaction. In a recent study, Rodriguez-Reyes et al. [35] demonstrated that noncovalent interactions between various oxygenates and their intermediates generated on the planar $\mathrm{Au}(111)$ model catalyst surface as well as their dispersive interactions with the gold surface can be closely linked to the reactivity of these species. An important factor in determining adsorption site preferences is the adsorbate surface coverage. As the distance between neighboring molecules decreases, intermolecular interactions may become large enough to strongly influence the adsorption energies and adsorption geometries [36]. This becomes particularly important for adsorbates with some degree of conformational flexibility. A density functional study by Verwüster et al. [36] demonstrated that the tilting angle of two substituted 4-mercapto-biphenylthiols changes linearly as a function of their surface coverages on the $\mathrm{Au}(111)$ surface. In another related work, Lee et al. [31] showed that at saturation surface coverages of $n$-butane on coinage materials, intermolecular interaction may account for up to $25 \%$ of the total adsorption energy. Furthermore, adsorbate chain length may have a direct effect on the adsorption energy due to the differences in dispersion interactions. For instance, the desorption energies of alkanes on $\mathrm{Au}(111)$ have been found to increase with increasing chain length $[37,38]$. It should further be noted that adsorption of organic molecules on Au and other metals may be influenced by several other factors such as polarity of the adsorbates [35], donor/acceptor character of the substituents [36] and intramolecular H-bonds [39].

The reason for the high selectivity of the gold catalysts in alcohol partial oxidation and oxidative coupling reactions can be related to the relatively low desorption energies of the reaction intermediates, which kinetically limit the surface residence times and further oxidation of these intermediates to thermodynamically more stable total oxidation products such as $\mathrm{CO}_{2}$. In the current work, we present a comparative experimental and theoretical investigation of the adsorption/ desorption energies of various ubiquitously utilized alcohols (methanol $(\mathrm{MeOH})$, ethanol (EtOH), n-propanol ( $n$-PrOH)) and esters (methyl acetate (MA), ethyl acetate (EA), methyl formate (MF)), which can be obtained via oxidative coupling reactions of the aforementioned alcohols on the $\mathrm{Au}(111)$ planar model catalyst surface. The choice of this particular set of molecules allows for a systematic exploration of the effects of polarity, molecular size and adsorbate surface coverage on the adsorption/desorption energies. Due to the difficulty associated with mapping the experimental saturation coverage onto an exact number of molecules per surface unit cell, the effect of the adsorbate surface coverage was computationally investigated at two different values, namely 1/4 monolayer (ML) and 1/9 ML. In our current definition of surface coverage, $1 / 4$ and 1/9 ML correspond to a single molecule in a $2 \times 2$ and a $3 \times 3$ surface unit cell, respectively. The aim of the current study is to provide a detailed account of the effects of several parameters in determining the adsorption/desorption characteristics of alcohols and esters on the $\mathrm{Au}(111)$ surface, which are crucial in predicting the selectivity in partial oxidation mechanisms.

\section{Methods}

\subsection{Computational Details}

Dispersion-corrected density functional theory (DFT) based total energy calculations were performed using a 
plane wave basis set with the Perdew-Burke-Ernzerhof (PBE) functional [40] to describe electronic exchange and correlation. Ultrasoft pseudopotentials were used to represent the ionic cores and to describe electron-ion interactions. The weak interaction between the adsorbates and the surface was described using a nonlocal, self-consistent van der Waals correlation (vdW-DF [41]) implemented in the Quantum Espresso code suite [42] via the Soler algorithm [43]. This van der Waals augmented PBE scheme was previously shown to work well in systems involving physisorption of organic molecules on metal surfaces [30]. The Au(111) surface was represented by $2 \times 2$ and $3 \times 3$ slabs for the two surface coverages $(1 / 4$ and 1/9 ML, respectively) with a vacuum of at least $20 \AA$ between periodic images in the direction perpendicular to the surface. Converged values for adsorption energies are obtained with slabs consisting of four atomic layers, with the two bottom layers fixed to mimic bulk behavior. Monkhorst-Pack [44] meshes of $12 \times 12 \times 1$ and $8 \times 8 \times 1$ points were used for the $2 \times 2$ and $3 \times 3$ slabs, respectively in performing the Brillouin zone integrations. A kinetic energy cutoff of $40 \mathrm{eV}$ was imposed to truncate the plane wave basis set while a cutoff of $400 \mathrm{eV}$ was used for the augmentation charge. Cold smearing [45] was employed to assist in the integration process with a width of 0.01 Ryd. The equilibrium geometry of the gas-phase molecules were calculated using traditional PBE and vdWDF; both of which yielded results in good agreement with experimental values [46]. Geometric optimization was performed using the Broyden-Fletcher-Goldfarb-Shanno (BFGS) algorithm [47-50].

The equilibrium lattice constant of face-centered cubic Au at the vdW-DF level is $4.179 \AA$, which agrees well with the experimental value of $4.078 \AA$. The adsorption energy $\left(E_{b}\right)$ was calculated using the equation

$\mathrm{E}_{\mathrm{b}}=\left(\mathrm{E}_{\mathrm{surf}}+\mathrm{E}_{\mathrm{ads}}\right)-\mathrm{E}_{\mathrm{ads}+\text { surf }}$,

where $E_{\text {ads }+ \text { surf }}, E_{\text {surf }}$, and $E_{\text {ads }}$ denote the total energy of the system (surface slab plus adsorbate), the energy of the $\mathrm{Au}$ slab, and the energy of the gas-phase adsorbate respectively. The total energies of the gas phase molecules and the bare $\mathrm{Au}$ surface were also calculated using the same vdW-DF scheme, for the sake of consistency. In order to quantify and separate the effects of intermolecular versus molecule/substrate interactions, additional calculations were performed where the adsorbate overlayer (at the minimum-energy configuration determined for the surface slab plus the adsorbate) was removed from the underlying Au substrate and subjected to a single self-consistent field (SCF) energy calculation using the same calculation parameters. The intermolecular interaction contribution to the adsorption energy was then calculated using the following equation:
$\mathrm{E}_{\text {int }}=\mathrm{E}_{\mathrm{ads}}-\mathrm{E}_{\mathrm{OL}}$

where $\mathrm{E}_{\mathrm{OL}}$ is the SCF energy of the isolated adsorbate overlayer while $\mathrm{E}_{\mathrm{ads}}$ is defined in Eq. (1). According to this prescription, a negative $E_{\text {int }}$ indicates repulsive interaction between the adsorbates while a positive value translates to intermolecular attraction. In an attempt to relate the observed trend in adsorption/desorption energies to molecular properties of the isolated species, the dipole moment and the isotropic polarizability of the gas phase oxygenates under investigation were also calculated in the DFT framework using the GAUSSIAN (G09) software [51] with the hybrid B3LYP exchange-correlation functional [51-54] and the aug-cc-pVTZ basis set [55].

\subsection{Experimental}

Experiments were performed in a custom-made multitechnique ultrahigh vacuum (UHV) surface analysis chamber with a base pressure of $2 \times 10^{-10}$ Torr. The UHV chamber was equipped with a temperature-programmed desorption (TPD) system utilizing a quadruple mass spectrometer (QMS, Dycor model DM200 M), an X-ray photoelectron spectrometer (XPS, Riber Mg/Al Dual anode and Riber Model EA 150 Electron Energy Analyzer), an Infrared Reflection Absorption Spectrometer (IRAS, Bruker Tensor 37 with custom-made external IR optics) and a custom-made rear-view low-energy electron diffraction (LEED) setup. An Au(111) single crystal disc (10 mm diameter, $2 \mathrm{~mm}$ thickness, both sides atomically polished, MaTeck $\mathrm{GmbH}$ ) was used as the substrate and was mounted on Ta wires, which can be resistively heated up to $1000 \mathrm{~K}$. The sample was cooled to ca. $90 \mathrm{~K}$ with liquid nitrogen and its temperature was monitored via a K-type thermocouple $(0.05 \mathrm{~mm}$, Omega) spot-welded on the lateral edge of the single crystal. During the TPD experiments, the sample was heated at a rate of $1 \mathrm{~K} / \mathrm{s}$ using a PID controller and a DC power supply (Heatwave Model 101303, Heatwave Inc.). Before the experiments, the $\mathrm{Au}(111)$ surface was cleaned by multiple cycles of $\mathrm{Ar}^{+}$ sputtering using an ion gun (LK technologies, NGI3000) operated with an accelerating voltage of $1.5 \mathrm{kV}$ and subsequent annealing to $873 \mathrm{~K}$ in vacuum. The cleanness of the $\mathrm{Au}(111)$ surface was confirmed by XPS and LEED. The investigated oxygenates were dosed onto the $\mathrm{Au}(111)$ surface through a high precision leak valve at $90 \mathrm{~K}$ and adsorbate surface coverages are reported in monolayer equivalents (MLE). Here, 1 MLE corresponds to the experimental saturation coverage of the first adsorbate overlayer on clean $\mathrm{Au}(111)$ surface. Methanol (99.9\%, purity), ethanol (99.8\%, purity), $n$-propanol (99\%, purity) methyl formate (99\%, purity), methyl acetate $(99.8 \%$, purity), and ethyl acetate (99.7\%, purity) were purchased 
from Sigma-Aldrich and were further purified via freezepump-thaw cycles.

\section{Results and Discussion}

Due to the weak interaction between the $\mathrm{Au}(111)$ surface and the oxygenate molecules investigated in the current work, the relevant potential energy surfaces (PES) are expected to reveal several closely spaced, shallow minima $[30,56]$. As it is computationally prohibitive to fully explore the PES and locate the global minimum, in our DFT investigation a restricted number of initial configurations were identified and subjected to geometry optimization. The alcohols were initially placed at an on-top location, with the oxygen of the hydroxyl group directly above a surface $\mathrm{Au}$ atom. The esters were instead initially positioned such that the $\mathrm{O}$ atom of the $-\mathrm{C}-\mathrm{O}-\mathrm{C}-$ functionality is in correspondence of a bridge site on the surface. These initial locations were previously identified as preferred adsorption sites for a subset of the adsorbates [57-62]. Parallel and perpendicular initial orientations were considered for both coverages. Upon structural optimization, at the lower coverage of 1/9 ML, the adsorbates were observed to experience only small deviations from their initial configurations. On the other hand, at the higher coverage of $1 / 4 \mathrm{ML}$, the optimized adsorption configurations were found to deviate significantly from the starting geometries. In particular, conformationally flexible molecules such as MA and EA were observed to undergo large reorientations and structural rearrangements. Initially parallel geometries reoriented in tilted configurations with respect to the surface while initially perpendicular geometries reoriented themselves nearly parallel to the gold surface. This indicates that at this high coverage, intermolecular interactions play a key role in determining the final adsorption geometry, and can potentially affect the relative stability of the adsorbates, as shall be discussed further in the text.

Figures 1 and 2 display the optimized adsorption geometries of the currently investigated alcohols and esters for the 1/9 ML coverage respectively. The $\mathrm{Au}-\mathrm{O}$ and $\mathrm{Au}-$ $\mathrm{H}$ distances reported in the figures represent the shortest distances from the adsorbates to the $\mathrm{Au}(111)$ surface.
Fig. 1 Adsorption geometries of $\mathrm{CH}_{3} \mathrm{OH}, \mathrm{CH}_{3} \mathrm{CH}_{2} \mathrm{OH}$ and $\mathrm{CH}_{3} \mathrm{CH}_{2} \mathrm{CH}_{2} \mathrm{OH}$ on $\mathrm{Au}(111)$ for the $1 / 9 \mathrm{ML}$ surface coverage in parallel (top panels) and perpendicular (middle panels) orientations. Side views for perpendicular adsorption geometries are shown on the bottom panels. The shortest distances between the molecules and the surface are also reported
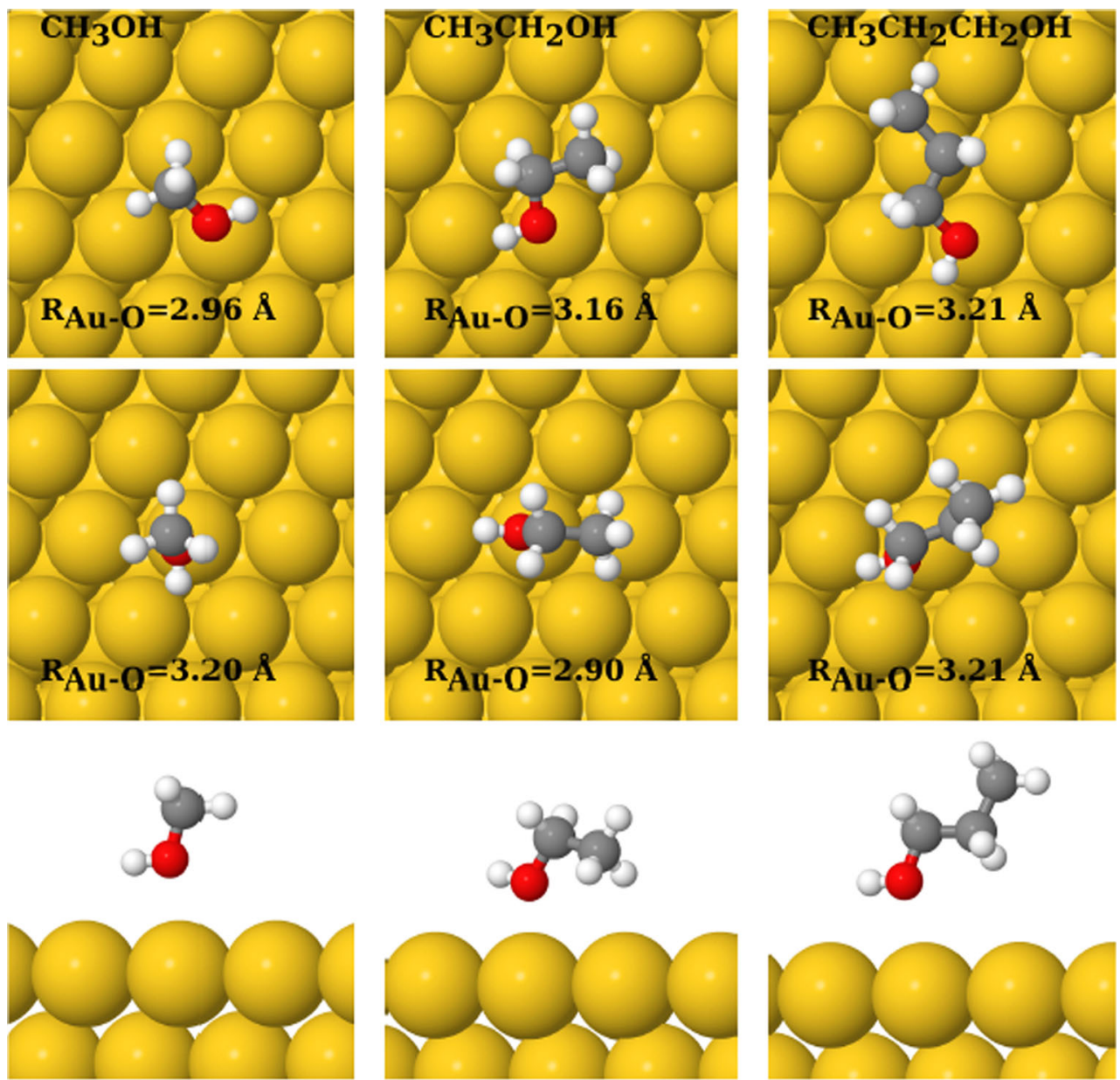
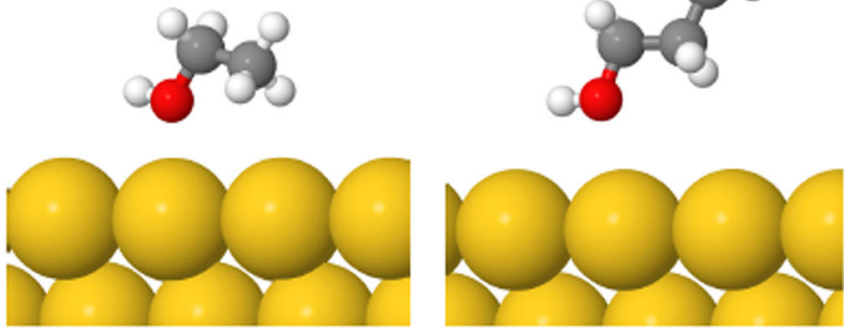
Fig. 2 Adsorption geometries of $\mathrm{HCOOCH}_{3}, \mathrm{CH}_{3} \mathrm{COOCH}_{3}$ and $\mathrm{CH}_{3} \mathrm{COOCH}_{2} \mathrm{CH}_{3}$ on $\mathrm{Au}(111)$ for the $1 / 9 \mathrm{ML}$ surface coverage in parallel (top panels) and perpendicular orientations (middle panels). Side views for perpendicular adsorption geometries are shown on the bottom panels. The shortest distances between the molecules and the surface are also reported
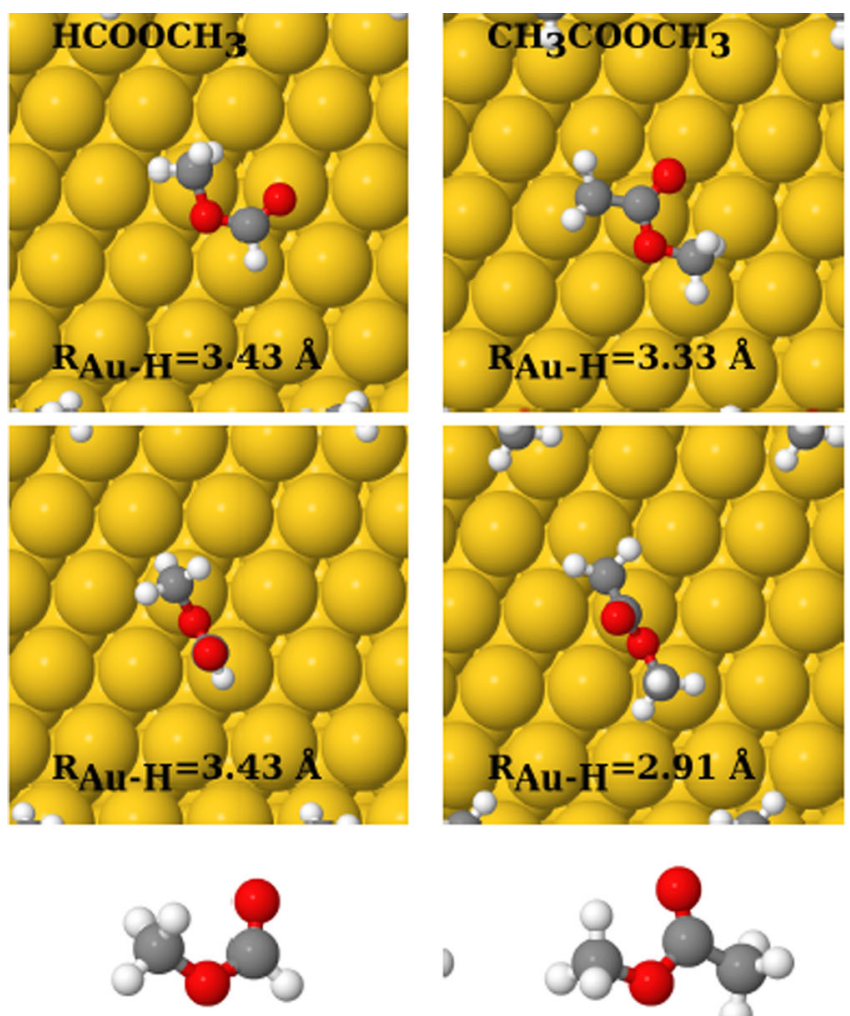

)
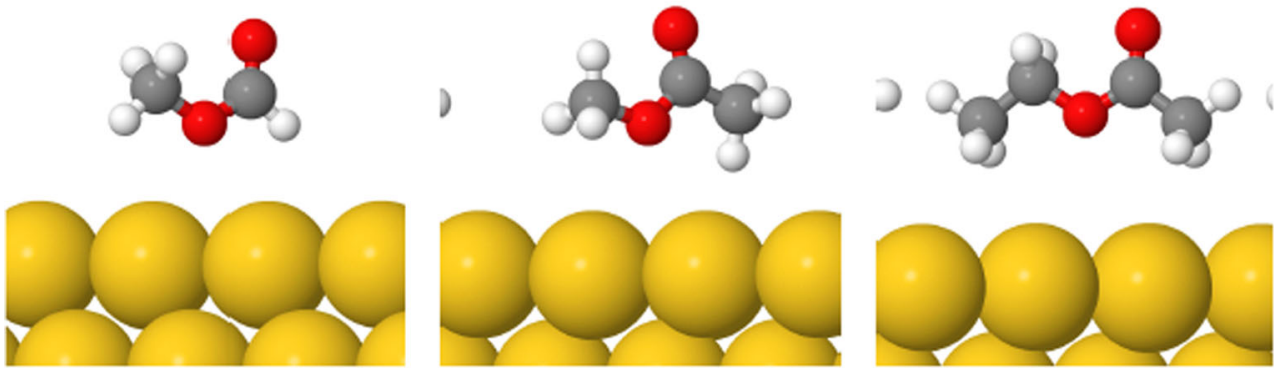

Table 1 lists the calculated adsorption energies of the investigated oxygenates where the lateral intermolecular interaction contributions are given in parentheses. For the 1/4 ML adsorbate coverage, we only report adsorption energies of parallel configurations in Table 1 , since the initially perpendicular configurations relax to parallel configurations as mentioned above, and displayed in Fig. 3. The calculated isotropic polarizabilities $(1 / 3$ of the trace of the polarizability tensor) and dipole moments of the gas phase oxygenates are instead collected in Table 2.

We first focus on the vdW-DF results for the 1/9 ML coverage. For all of the alcohols and the esters considered, adsorption energies for the parallel configurations increase monotonically with increasing molecular weight. This trend is also generally observed for the perpendicular orientation of the adsorbates, with the exception of $\mathrm{EtOH}$ and $\mathrm{n}-\mathrm{PrOH}$, for which a similar stability is predicted. Furthermore, perpendicular adsorption geometries are found to be relatively less stable than the corresponding parallel configurations by ca. $5.0-15.0 \mathrm{~kJ} / \mathrm{mol}(\sim 50-150 \mathrm{meV})$. At this relatively low coverage, the lateral adsorbate-adsorbate interactions play only a minor role, and the energetics of adsorption is determined to a large extent by the affinity of a single and a rather isolated adsorbate molecule to the surface.

Intermolecular interactions are, on the other hand, not negligible at the higher coverage of $1 / 4 \mathrm{ML}$, and tend to stabilize adsorption geometries that are markedly different from those observed at the lower 1/9 ML coverage. For all three alcohols, the adsorption configuration is a tilted geometry with the $\mathrm{O}$ atom pointing towards a surface $\mathrm{Au}$ atom. For $n-\mathrm{PrOH}$, geometry relaxation involves a rotation in the plane parallel to the surface and slight distortions of the molecular backbone. MA relaxes through rotations of the $\mathrm{CH}_{3}$ groups around the $\mathrm{C}-\mathrm{C}$ and $\mathrm{C}-\mathrm{O}$ bonds while in the case of the more flexible EA, the configuration of the adsorbed species at the higher coverage strongly deviates from that of the isolated gas-phase species. It is worth mentioning that trends observed for the magnitude of the intermolecular interactions at the higher coverage of $1 / 4$ ML are parallel to the polarizability trends presented in Table 2, revealing the significance of dispersion interactions which tend to increase with increasing polarizability. On the other hand, such a monotonic trend between the 
Table 1 Calculated adsorption energies (in $\mathrm{kJ} / \mathrm{mol}$ ) of the oxygenates on $\mathrm{Au}(111)$ for $1 / 4$ and $1 / 9 \mathrm{ML}$ surface coverages along with the experimental TPD adsorption/desorption energies at saturation coverage. The numbers in parentheses correspond to the contribution of the intermolecular interactions to the total computed adsorption energies. Negative values correspond to repulsive interactions

\begin{tabular}{lllll}
\hline & \multicolumn{2}{l}{ DFT-DF 1/9 ML } & DFT-DF 1/4 ML & TPD \\
\cline { 2 - 3 } & Parallel & Perpendicular & Parallel & 40.4 \\
\hline $\mathrm{MeOH}$ & $34.5(0.4)$ & $25.7(2.0)$ & $36.3(7.2)$ & $49.8,45.8^{\mathrm{b}}$ \\
$\mathrm{EtOH}$ & $42.2(0.3)$ & $37.1(-0.9)$ & $50.3(13.3)$ & 51.7 \\
$n-\mathrm{PrOH}$ & $49.3(-1.3)$ & $35.2(-2.0)$ & $61.3(18.4)$ & 38.2 \\
$\mathrm{MF}$ & $37.0(-0.8)$ & $30.4(1.9)$ & $47.8(12.9)$ & 43.6 \\
$\mathrm{MA}$ & $48.1(5.8)$ & $38.2(0.2)$ & $56.6(21.3)$ & 49.5 \\
EA & $60.0(4.9)$ & $46.3(2.6)$ & $61.5(25.7)$ &
\end{tabular}

${ }^{a}$ Experimental desorption energies were calculated using the Redhead method [65] utilizing the corresponding $\mathrm{T}_{\max }$ values observed for the first monolayer in the TPD data

b An alternative value of $45.8 \mathrm{~kJ} / \mathrm{mol}$ can also be derived from the TPD data reported in [11, 26] revealing a EtOH/Au(111) monolayer desorption maximum at $175 \mathrm{~K}$

calculated dipole moments and corresponding intermolecular interactions are not visible.

Desorption energies from the $\mathrm{Au}(111)$ surface were also studied experimentally by employing the TPD technique. Consistency between the mass spectroscopic fragmentation patterns of the investigated molecules before dosing the oxygenates onto the $\mathrm{Au}(111)$ surface and that of the desorbing species clearly indicates that all of the currently investigated molecules adsorb/desorb in a molecular (i.e. non-dissociative) fashion. TPD spectra of methanol adsorbed on clean $\mathrm{Au}(111)$ surface are shown in Fig. 4a. The spectra presented here are in very good agreement with the results provided in a former report [63]. The desorption maximum located at $155 \mathrm{~K}$ reveals 1 st order desorption kinetics and corresponds to the methanol molecules on the first monolayer of the overlayer that is in direct contact with the $\mathrm{Au}(111)$ surface. Convergence of the intensity of this desorption feature with increasing $\mathrm{MeOH}$ exposures indicates the saturation of the first adsorbate overlayer. Integrated desorption signal of this saturated TPD peak was used for the experimental estimation of the adsorbate surface coverage and this integrated signal was assigned to be 1 MLE. It can be noted that the desorption signals located within $120-150 \mathrm{~K}$ do not converge to a saturation value, as these weakly-bound states are associated with multilayer adsorption. It is worth mentioning that the coverages in the TPD experiments cannot be directly compared to coverages used in the current DFT calculations. In the TPD experiments, 1 MLE adsorbate coverage corresponds to the experimental saturation coverage of the first adsorbate overlayer observed on the $\mathrm{Au}(111)$ surface before the appearance of the second adsorbate overlayer (i.e. corresponding to less than an adsorbate per surface atom); while in the DFT calculations, $1 \mathrm{ML}$ surface coverage is defined as the number of adsorbate molecules per surface Au atom on $\mathrm{Au}(111)$.

These general desorption characteristics are also valid for the EtOH adsorption on $\mathrm{Au}(111)$, shown in Fig. 4b. It is seen that the first monolayer desorption maximum for the EtOH/ $\mathrm{Au}(111)$ appears at a higher temperature (i.e. $190 \mathrm{~K}$ ) than that of the $\mathrm{MeOH} / \mathrm{Au}(111)$ case. As mentioned in the footnote of Table 1 , the $\mathrm{T}_{\max }$ value measured in the current experiments for the first monolayer of the EtOH/Au(111) adsorption system is slightly higher than that of a previous measurement in the literature $(175 \mathrm{~K})[11,26]$. This minor discrepancy can be associated with a contribution from background $\mathrm{H}_{2} \mathrm{O}$ adsorption/desorption $(\mathrm{m} / \mathrm{z}=18)$ which also reveals itself as a minor ethanol desorption peak (tentatively associated with the desorption of the $\mathrm{C}_{2} \mathrm{H}_{5} \mathrm{OH} \cdot x \mathrm{H}_{2} \mathrm{O}$ phase) at $150 \mathrm{~K}$ accompanied by water desorption at the same temperature. In the current experiments, we utilized EtOH with a purity greater than $99.8 \%$, which was purified further with freeze-pump-thaw cycles. However, even after this purification protocol, the presence of a minor amount of water can still be detected at high ethanol coverages due to the high sensitivity of the TPD technique. Furthermore, desorption of the EtOH multilayers were observed in the range of $160-180 \mathrm{~K}$.

In an analogous fashion, TPD spectra of $n$-PrOH desorbing from the $\mathrm{Au}(111)$ surface are presented in Fig. 4c and are in very good agreement with the literature [64]. The monolayer desorption peak of $n$-PrOH located at $197 \mathrm{~K}$ also demonstrates first-order desorption kinetics as in the case of $\mathrm{MeOH} / \mathrm{Au}(111)$ and $\mathrm{EtOH} / \mathrm{Au}(111)$ systems.

In a subsequent set of TPD experiments, industrially relevant products of the alcohol oxidative coupling reactions (i.e. MF, MA, EA) were investigated in a comparative manner. TPD spectra of the corresponding experiments are 
Fig. 3 Adsorption geometries (top and side views) of $\mathrm{CH}_{3} \mathrm{OH}$, $\mathrm{CH}_{3} \mathrm{CH}_{2} \mathrm{OH}, \mathrm{CH}_{3} \mathrm{CH}_{2} \mathrm{CH}_{2} \mathrm{OH}$, $\mathrm{HCOOCH}_{3}, \mathrm{CH}_{3} \mathrm{COOCH}_{3}$ and $\mathrm{CH}_{3} \mathrm{COOCH}_{2} \mathrm{CH}_{3}$ on $\mathrm{Au}(111)$ for the 1/4 ML coverage. The shortest distances between the molecules and the surface are also reported in the figure
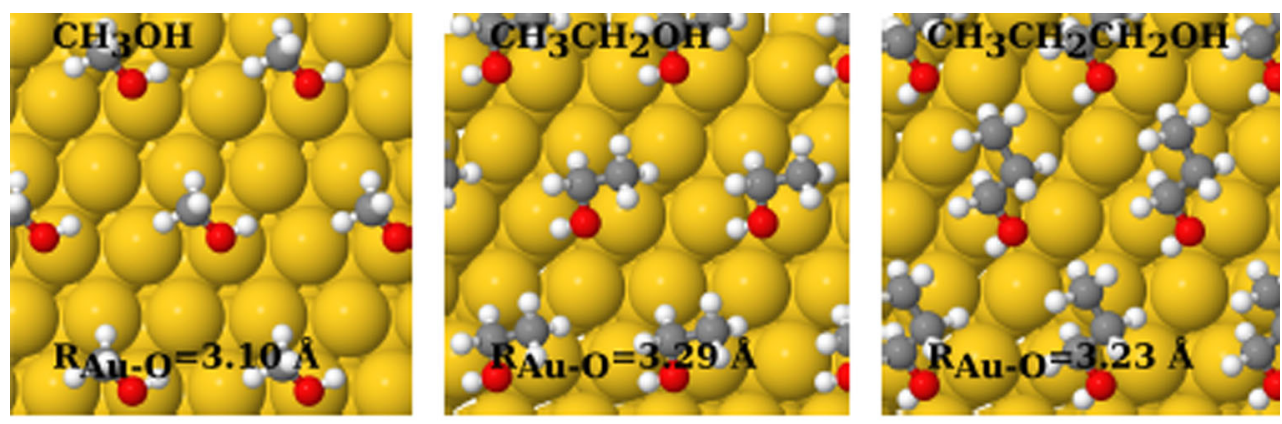

3
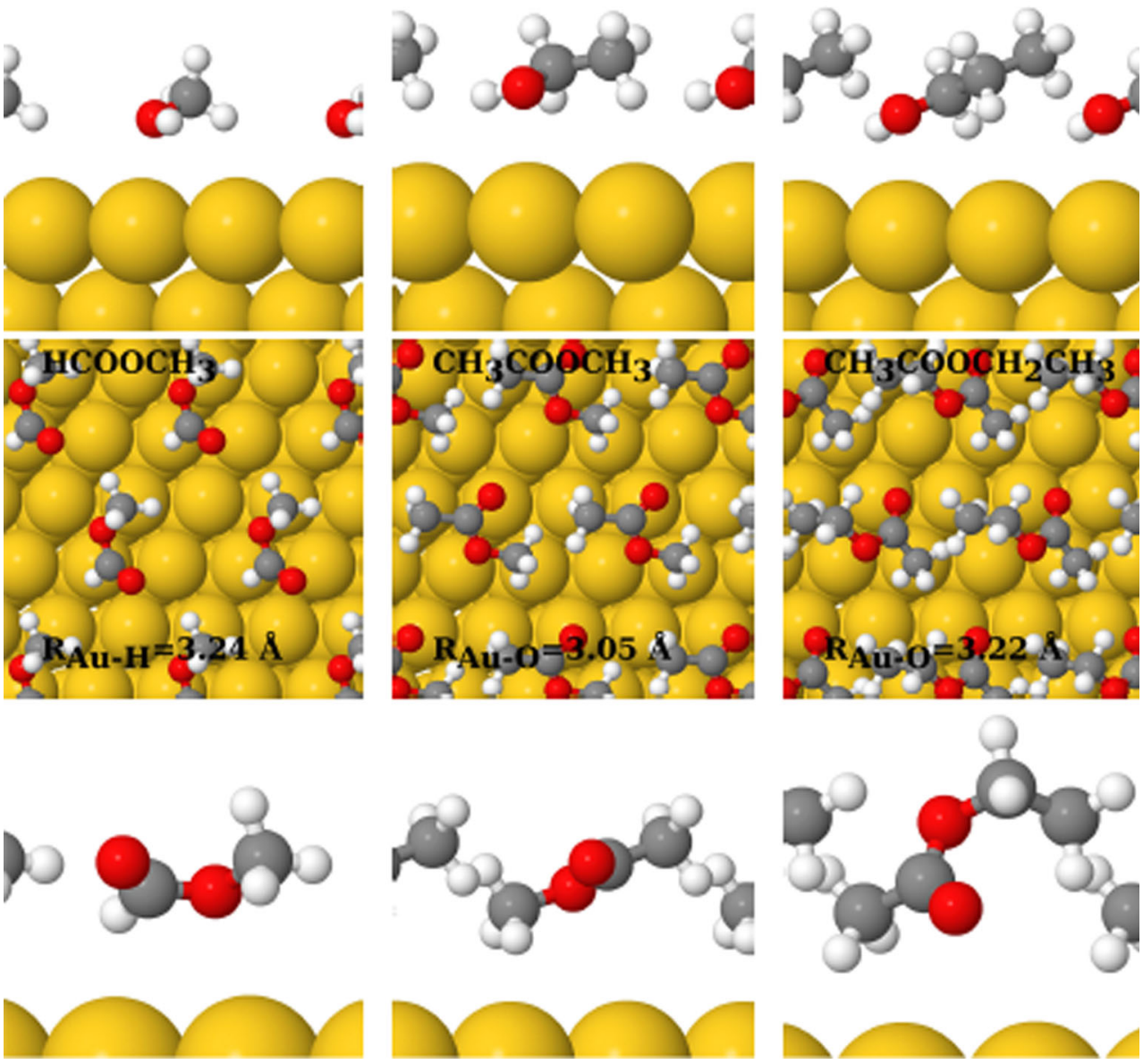

Table 2 B3LYP/aug-cc-pVTZ isotropic polarizabilities, $\alpha$, and dipole moments, $\mu$, of the gas phase molecules

\begin{tabular}{lll}
\hline & $\alpha\left(\AA^{3}\right)$ & $\mu(\mathrm{D})$ \\
\hline $\mathrm{MeOH}$ & 3.20 & 1.66 \\
$\mathrm{EtOH}$ & 5.33 & 1.58 \\
$n-\mathrm{PrOH}$ & 7.23 & 1.48 \\
$\mathrm{MF}$ & 5.12 & 1.97 \\
$\mathrm{MA}$ & 6.92 & 1.92 \\
$\mathrm{EA}$ & 8.80 & 2.11 \\
\hline
\end{tabular}

shown in Fig. 5. As in the case of alcohols, all esters were found to adsorb/desorb on/from the Au(111) planar model catalyst surface in a reversible and molecular fashion without any indication of dissociation. The first-monolayer desorption peaks for MF, MA and EA were observed at 147, 167, and $189 \mathrm{~K}$; respectively. Unsaturated desorption peaks seen in Fig. 5 at lower temperatures are associated with multilayer desorption states revealing zeroth-order desorption kinetics.

Redhead method [65] can be used to calculate the desorption energies of the adsorbates originating from the first monolayer on $\mathrm{Au}(111)$ following first-order desorption kinetics. Corresponding desorption energies $\left(\Delta E_{d e s}\right)$ can be estimated using Eq. (3) given below [66]:

$\Delta E_{d e s}=R T_{\max }\left[\ln \frac{v_{1} T_{\max }}{\beta}-\ln \frac{\Delta E_{\text {des }}}{R T_{\max }}\right]$, 
Fig. 4 TPD spectra corresponding to increasing surface coverages of a $\mathrm{CH}_{3} \mathrm{OH}$, b $\mathrm{CH}_{3} \mathrm{CH}_{2} \mathrm{OH}$, and c $\mathrm{CH}_{3} \mathrm{CH}_{2} \mathrm{CH}_{2} \mathrm{OH}$ dosed on clean $\mathrm{Au}(111)$ at $90 \mathrm{~K}$
Fig. 5 TPD spectra corresponding to increasing surface coverages of a $\mathrm{HCOOCH}_{3}$, b $\mathrm{CH}_{3} \mathrm{COOCH}_{3}$, and $\mathbf{c} \mathrm{CH}_{3} \mathrm{COOCH}_{2} \mathrm{CH}_{3}$ dosed on clean $\mathrm{Au}(111)$ at $90 \mathrm{~K}$
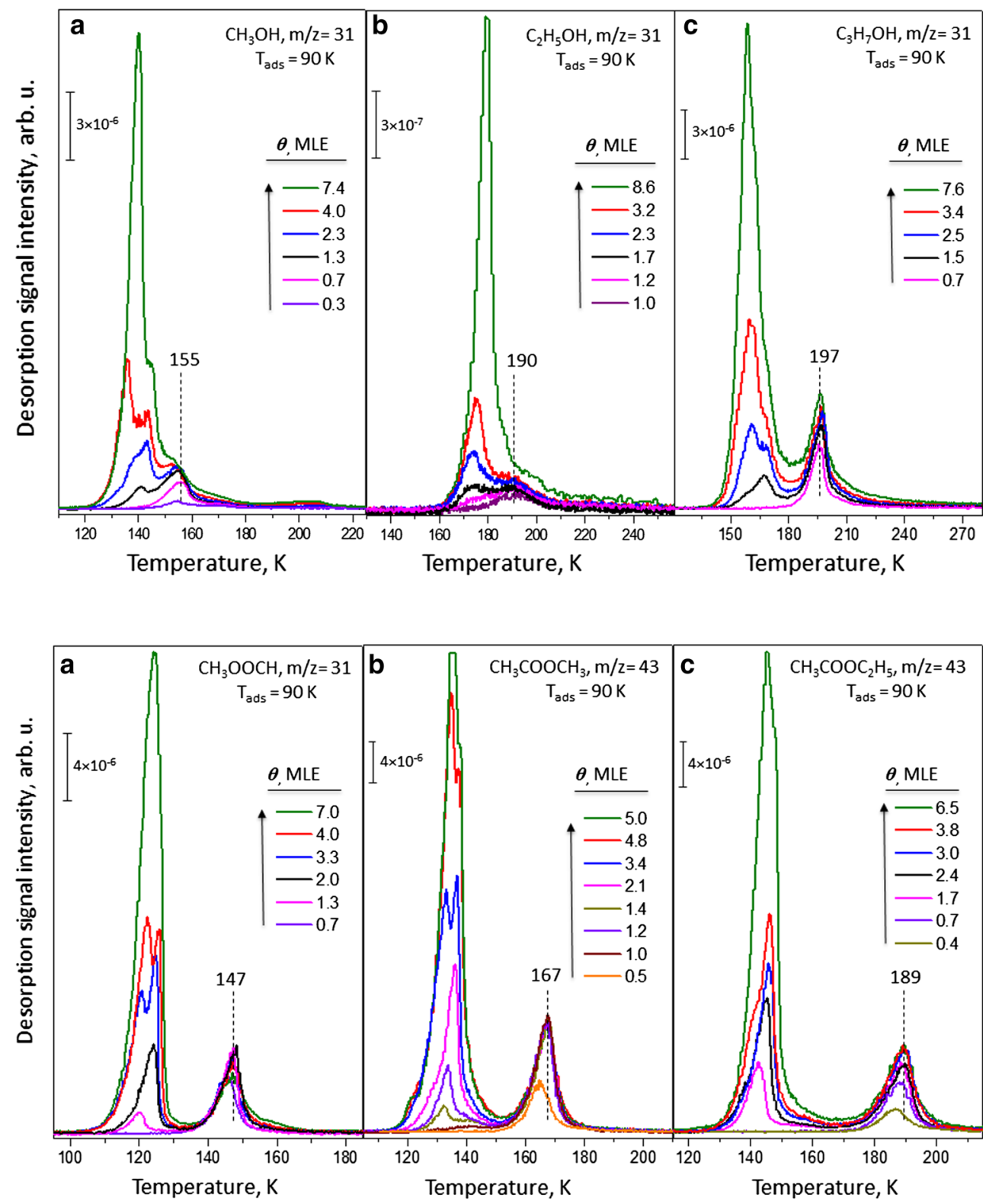

where $T_{\text {max }}$ is the temperature maximum of the 1 st order desorption peak, and $\beta$ is the heating rate $(1 \mathrm{~K} / \mathrm{s})$. The preexponential factor $v_{1}$ in the current calculations is estimated to be $10^{13} \mathrm{~s}^{-1}$. The last term in the right hand side of Eq. (3) (i.e. $\ln \frac{\Delta E_{\text {des }}}{R T_{\max }}$ ) was approximated to be 3.64 [66]. Note that for very low adsorbate coverages, experimentally observed TPD profiles given in Figs. 4 and 5 reveal a minor high-temperature tail which is most likely due to the preferential adsorption on surface defects rendering themselves as strong adsorption sites. These strong adsorption (i.e. defect) sites are not representative of the regular $\mathrm{Au}(111)$ terraces. Hence for the further analysis of the experimental TPD results via Redhead analysis, we utilized desorption maxima corresponding to the higher coverages of the first adsorption layer revealing first-order desorption kinetics.

Figure 6 is a concise comparison of our theoretical adsorption energies for $1 / 9$ and 1/4 ML coverages with experimental desorption energies. In spite of the difficulties associated with matching experimental surface coverages to those theoretically imposed, we find good agreement between the DFT and TPD results. An exception to this is EA, for which the DFT adsorption energy overestimates the TPD value for both coverages. For the smallest alcohols ( $\mathrm{MeOH}$ and $\mathrm{EtOH})$, the DFT adsorption energies for the 1/4 ML coverage are in better accord with the TPD results while for the remaining, larger molecules, the adsorption energies calculated for the 1/9 ML coverage are closer to 


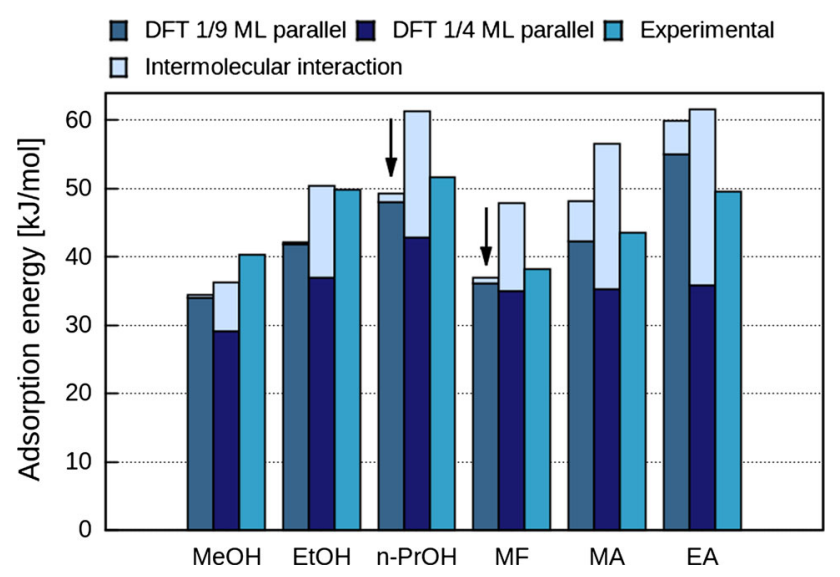

Fig. 6 Adsorption energies of the investigated oxygenates on the $\mathrm{Au}(111)$ model catalyst surface for the 1/9 ML parallel (left columns) and 1/4 ML parallel (middle columns) configurations and the corresponding experimental values (right columns). The top portion of the bars represents the intermolecular interaction contribution to the adsorption energy. Downward arrows indicate those cases where the intermolecular interaction energy is negative

the experimental values. This is consistent with the expectation that the saturation coverage in the experiments for the smaller molecules are more likely to correspond to a larger number of molecules per surface unit cell.

At large separations, the noncovalent interactions between neutral systems are dominated by electrostatic and dispersion forces. The dispersive portion can only be correctly handled by exchange-correlation functionals with the correct long-range behavior such as DFT-DF [41]. According to classical point-dipole models for the adsorption of polar molecules, lateral interactions are repulsive in the case of perpendicular orientation with respect to the underlying surface and attractive when the orientation is parallel. In the case of tilted dipoles, the sense of the interaction depends on the tilt angle [67]. While the lateral dipole-dipole interaction in the pointdipole model has been demonstrated to match PBE adsorption energies for various polar molecules for intermolecular separations as small as $2 \AA$, the adlayer-surface interaction presents difficulties, including the ambiguity associated with choosing the mirror plane [67, 68]. Nonetheless, the model agrees with PBE results within a few meV for molecule-distance separations as small as $4 \AA$ [68]. Even if for polarizable dipoles, the separation between dipole-dipole and dipole-image dipole contributions is less clear-cut, we used a separate set of single-point PBE calculations using the DFT-DF optimized geometries of the adsorbed overlayers in an attempt to estimate the relative contributions of each of the two electrostatic terms. An inspection of the intermolecular interaction contributions and adhesion energies of the overlayers reveals that for all species considered (with the exception of MA and
EA in the parallel adsorption configuration), the lateral interaction is repulsive in the $1 / 9 \mathrm{ML}$ coverage. For the $1 / 4$ ML coverage, on the other hand, the interaction is weak but attractive. This indicates that a large portion of the mostly attractive lateral interaction between the molecules is due to dispersion. The electrostatic adlayer-surface interaction is instead attractive for all cases and is largely independent of coverage. Moreover, even if the adhesion energy is significantly larger for the alcohols compared to the esters, the largest contribution to interaction is due to dispersion rather than electrostatics.

A general assessment of the relative experimental and theoretical desorption energies of esters (i.e. products of oxidative coupling reactions) in comparison to the corresponding alcohols suggests that the esters have similar or even slightly lower desorption energies than their relevant alcohols. This is an important finding in the context of catalyst selectivity and may, in part, be due to the weaker electrostatic surface-adsorbate interaction calculated for esters.

For all molecules considered, the calculated adsorption energies are greater for the $1 / 4 \mathrm{ML}$ coverage compared to $1 / 9$ ML. This is also supported by the TPD data given in Fig. 5 revealing that the $\mathrm{T}_{\max }$ values for lower coverages are ca. $3 \mathrm{~K}$ lower than the $\mathrm{T}_{\max }$ values at the saturation of the first monolayer, suggesting the strengthening of the adsorption energy with increasing coverage.

The increase observed in the intermolecular interaction (top portion in Fig. 6) for the 1/4 ML coverage with increasing chain length for both alcohols and esters is mostly due to dispersion interactions as the lateral dipoledipole interactions are small. The underlying reasons are expected to be decreasing intermolecular distance and increasing polarizability with increasing molecular size. The intermolecular interactions currently computed can exceed one-third of the total adsorption energy at the 1/4 ML adsorbate coverage.

Figure 6 further suggests that, for all molecules, when the lateral interaction component is disregarded, the 1/4 ML coverage yields slightly smaller adsorption energies than those calculated for the 1/9 ML coverage (the lower portions of the columns in Fig. 6). This can possibly be related to the observation that the contact area between the molecules and the surface is smaller in the higher coverage due to rotation of the molecules into a tilted configuration, reducing the overlap of electronic densities. It is thus apparent that disregarding intermolecular interactions may lead to discernable changes in the rankings of the computationally obtained relative adsorption strengths. For instance, without the contribution of intermolecular interactions at the 1/4 ML adsorbate coverage, computationally calculated adsorption energies are almost the same $(\sim 35 \mathrm{~kJ} / \mathrm{mol})$ for $\mathrm{MF}$, MA and $\mathrm{EA}$, while this trend 
changes significantly when the energy of intermolecular interactions are included.

The contribution of the interaction between the surface and the molecule to the total adsorption energy of the species reveal a well-defined trend for the lower coverage of 1/9 ML. At this coverage, the intermolecular interactions are minimal and therefore the adsorption energy directly reflects the magnitude of the molecule-surface interaction. For all alcohols and esters, the surface-molecule interaction increases as a function of chain length. As discussed above, the electrostatic contribution to this energy for alcohols may be significant. However, this contribution appears to decrease with increasing molecular size and therefore the contribution setting this trend is then once again dispersive forces.

\section{Conclusion}

In the current work, $\mathrm{Au}(111)$ single crystal surface was used to study the adsorption behavior of alcohols and esters in a comparative manner via TPD experiments and DFT calculations in association with oxidative coupling reactions. All oxygenates used in the current study (i.e. $\mathrm{CH}_{3} \mathrm{OH}, \mathrm{CH}_{3}$ $\mathrm{CH}_{2} \mathrm{OH}, \mathrm{CH}_{3} \mathrm{CH}_{2} \mathrm{CH}_{2} \mathrm{OH}, \mathrm{HCOOCH}_{3}, \mathrm{CH}_{3} \mathrm{COOCH}_{3}$ and $\mathrm{CH}_{3} \mathrm{COOCH}_{2} \mathrm{CH}_{3}$ ) were found to adsorb non-dissociatively on $\mathrm{Au}(111)$ at $90 \mathrm{~K}$ and desorb from the surface reversibly in a molecular fashion. The trends uncovered in the adsorption/desorption energies as a function of coverage and chain length were explained by identifying the contributions from the different flavors of van der Waals forces, which were proven to be crucial in the catalytic selectivity of the surface in partial oxidation processes. Experimental adsorption/desorption energies of the investigated esters are slightly lower than those of the alcohols from which they originate through oxidative coupling reactions. This could be an indication of the selectively of the $A u(111)$ surface, allowing facile removal of partial oxidation products immediately after their generation and preventing their complete oxidation to higher oxygenates.

Acknowledgments E. V., M. K., A. A. S. and E. O. acknowledge financial support from the Scientific and Technological Research Council of Turkey (TÜBITAK, Program Code: 2221 and Grant No: 112T589). H. U. and D. T. gratefully acknowledge the support by TÜBITAK Grant No. 113F099 in addition to the computational resources provided by the National Center of Academic Network and Information (TÜBITAK ULAKBIM) and the National Center for High Performance Computing (UHEM) Grant No. 5003342014.

\section{References}

1. Forouzani M, Mardani HR, Ziari M, Malekzadeh A, Biparva P (2015) Chem Eng J 275:220

2. Ahn SH, Liu Y, Moffat TP (2015) ACS Catal 5:2124-2136
3. Wang R, Wu Z, Wang G, Qin Z, Chen C, Dong M, Zhu H, Fan W, Wang J (2015) RSC Adv 5:44835-44839

4. Kannan R, Silva AA, Cardoso FM, Gupta G, Aslam Z, Sharma S, Steinberger Wilckens R (2015) RSC Adv 5:36993-36998

5. Garcia G, Stoffelsma C, Rodriguez P, Koper MTM (2015) Surf Sci 631:267-271

6. Xie R, Chen M, Wang J, Mei S, Pan Y, Gu H (2015) RSC Adv 5:650-653

7. Hao Y, Yang Y, Hong L, Yuan J, Niu L, Gui Y (2014) ACS Appl Mater Interfaces 6:21986-21994

8. Abdelouahab-Reddam Z, Mail RE, Coloma F, SepulvedaEscribano A (2015) Catal Today 249:109-116

9. Asgardi J, Calderon JC, Alcaide F, Querejeta A, Calvillo L, Lazaro MJ, Garcia G, Pastor E (2015) Appl Catal B 168-169:33-41

10. Gong J, Mullins CB (2008) J Am Chem Soc 130:16458-16459

11. Boronat M, Leyva-Perez A, Corma A (2014) Acc Chem Res 47:834-844

12. Liu X, He L, Liu Y-M (2014) and Y. Cao Acc Chem Res 47:793-804

13. Wittstock A, Baumer M (2014) Acc Chem Res 47:731-739

14. Xu B, Liu X, Haubrich J, Madix R, Friend CM (2009) Angew Chem Int Ed 48:4206-4209

15. Haruta M (1989) J Catal 115:301-309

16. Liu X, Xu B, Haubrich J, Madix RJ, Friend CM (2009) J Am Chem Soc 131:5757-5759

17. Liu X, Friend CM (2010) Langmuir 26:16552-16557

18. Bobuatong K, Karanjit S, Fukuda R, Ehara M, Sakurai H (2012) Phys Chem Chem Phys 14:3103-3111

19. Wang H, Wang C, Yan H, Yi H, Lu J (2015) J Catal 324:59-68

20. Wittstock A, Zielasek V, Biener J, Friend CM, Bäumer M (2010) Science 327:319-322

21. Karatok M, Vovk EI, Shah AA, Turksoy A, Ozensoy E (2015) Surf Sci 641:289-293

22. Outka DA, Madix RJ (1987) J Am Chem Soc 109:1708-1714

23. Lazaga MA, Wickham DT, Parker DH, Kastanas GN, Koel BE (1993) ACS Symp Ser 523:90-109

24. Outka DA, Madix RJ (1987) Surf Sci 179:361-376

25. Madix RJ, Friend CM, Liu XY (2008) J Catal 258:410-413

26. Syomin D, Koel BE (2002) Surf Sci 498:53-60

27. Pan M, Flaherty DW, Mullins CB (2011) J Phys Chem Lett 2:1363-1367

28. Davis KA, Goodman DW (2000) J Phys Chem B 104:8557-8562

29. Chesters MA, Somorjai GA (1975) Surf Sci 52:21-28

30. Carrasco J, Liu W, Michaelides A, Tkatchenko A (2014) J Chem Phys 140:084704

31. Lee K, Morikawa Y, Langreth DC (2010) Phys Rev B 82:155461

32. Buimaga-Iarinca L, Morari C (2014) Theoret Chem Acc 133: 1502

33. Bedolla PO, Feldbauer G, Wolloch M, Eder SJ, Drr N, Mohn P, Redinger J, Vernes A (2014) J Phys Chem C 118:17608-17615

34. Fajn J, Teixeira F, Gomes J, Cordeiro M (2015) Theor Chem Acc 134:67

35. Rodriguez-Reyes JCF, Siler CGF, Liu W, Tkatchenko A, Friend CM, Madix RJ (2014) J Am Chem Soc 136(13333-13340):19

36. Verwüster E, Hofmann OT, Egger DA, Zojer E (2015) J Phys Chem C 119:7817-7825

37. Wetterer SM, Lavrich DJ, Cummings T, Bernasek SL, Scoles G (1998) J Phys Chem B 102(46):9266-9275

38. Baxter RJ, Teobaldi G, Zerbetto F (2003) Langmuir 19:7335-7340

39. Garcia-Muelas R, Lopez N (2014) J Phys Chem C 118:17531-17537

40. Perdew J, Burke K, Ernzerhof M (1996) Phys Rev Lett 77:3865-3868

41. Dion M, Rydberg H, Schroder E, Langreth DC, Lundqvist BI (2004) Phys Rev Lett 92:246401 
42. Giannozzi P, Baroni S, Bonini N, Calandra M, Car R, Cavazzoni C, Ceresoli D, Chiarotti GL, Cococcioni M, Dabo I, Corso AD, de Gironcoli S, Fabris S, Fratesi G, Gebauer R, Gerstmann U, Gougoussis C, Kokalj A, Lazzeri M, Martin-Samos L, Marzari N, Mauri F, Mazzarello R, Paolini S, Pasquarello A, Paulatto L, Sbraccia C, Scandolo S, Sclauzero G, Seitsonen AP, Smogunov A, Umari P, Wentzcovitch RM (2009) J Phys: Condens Matter 21:395502

43. Roman-Perez G, Soler J (2009) Phys Rev Lett 103:096102

44. Monkhorst HJ, Pack JD (1976) Phys Rev B 13:5188-5192

45. Marzari N, Vanderbilt D, De Vita A, Payne MC (1999) Phys Rev Lett 82:3296-3299

46. NIST Computational Chemistry Comparison and Benchmark Database, NIST Standard Reference Database Number 101 Release 17b, September 2015, Editor: Russell D. Johnson III, http://cccbdb.nist.gov/

47. Broyden CG (1970) J Inst Math Appl 6:76-90

48. Fletcher R (1970) Computer Journal 13:317-322

49. Goldfarb D (1970) Mathematics of Computation 24:23-26

50. Shanno DF (1970) Math Comput 24:647-656

51. Gaussian 09, Revision E.01, Frisch MJ, Trucks GW, Schlegel HB, Scuseria GE, Robb MA, Cheeseman JR, Scalmani G, Barone V, Mennucci B, Petersson GA, Nakatsuji H, Caricato M, Li X, Hratchian HP, Izmaylov AF, Bloino J, Zheng G, Sonnenberg JL, Hada M, Ehara M, Toyota K, Fukuda R, Hasegawa J, Ishida M, Nakajima T, Honda Y, Kitao O, Nakai H, Vreven T, Montgomery JA Jr, Peralta JE, Ogliaro F, Bearpark M, Heyd JJ, Brothers E, Kudin KN, Staroverov VN, Kobayashi R, Normand J, Raghavachari K, Rendell A, Burant JC, Iyengar SS, Tomasi J, Cossi M, Rega N, Millam JM, Klene M, Knox JE, Cross JB, Bakken V, Adamo C, Jaramillo J, Gomperts R, Stratmann RE, Yazyev O, Austin AJ, Cammi R, Pomelli C, Ochterski JW, Martin RL, Morokuma K, Zakrzewski VG, Voth GA, Salvador P,
Dannenberg JJ, Dapprich S, Daniels AD, Farkas Ö, Foresman JB, Ortiz JV, Cioslowski J and D. J. Fox (2009), Gaussian Inc, Wallingford CT

52. Becke AD (1993) J Chem Phys 98:5648-5652

53. Lee C, Yang W, Parr RG (1988) Phys Rev B 37:785-789

54. Stephens PJ, Devlin FJ, Chabalowski CF, Frisch MJ (1994) J Phys Chem 98:11623-11627

55. Kendall RA, Dunning TH Jr, Harrison RJ (1992) J Chem Phys 96:6796-6806

56. Sayin CS, Toffoli D, Ustunel H (2015) Appl Surf Sci 351:344-352

57. Wang L, He C, Zhang W, Li Z, Yang J (2014) J Phys Chem C 118:17511-17520

58. Chang C-R, Yang X-F, Long B, Li J (2013) ACS Catal 3:1693-1699

59. Xu B, Haubrich J, Baker TA, Kaxiras E, Friend CM (2011) J Phys Chem C 115:3703-3708

60. Liu S, Jin P, Zhang D, Hao C, Yang X (2013) Appl Surf Sci 265:443-451

61. Meng Q, Shena Y, Xu J, Ma X, Gong J (2012) Surf Sci 606:1608-1617

62. Fartaria RPS, Freitas FFM, Silva FMS (2007) Fernandes. Int J Quantum Chem 107:2169-2177

63. Gong J, Flaherty DW, Ojifinni RA, White JM, Mullins CB (2008) Chem Phys Chem 112:5501-5509

64. Gong J, Flaherty DW, Yan T, Mullins CB (2008) Chem Phys Chem 9:2461-2466

65. Redhead PA (1962) Vacuum 12:203-211

66. Niemantsverdriet, J.W. Spectroscopy in Catalysis: An Introduction, Third Edition, Wiley-VCH; (July 23, 2007)

67. Kokalj T (2011) Phys Rev B 84:045418

68. Fernandez-Torre D, Kupiainen O, Pyykko P, Halonen L (2009) Chem Phys Lett 471:239-243 